\title{
Stroboscopic visual training improves information encoding in short-term memory
}

\author{
L. Gregory Appelbaum - Matthew S. Cain \\ Julia E. Schroeder • Elise F. Darling • Stephen R. Mitroff
}

Published online: 19 July 2012

(C) Psychonomic Society, Inc. 2012

\begin{abstract}
The visual system has developed to transform an undifferentiated and continuous flow of information into discrete and manageable representations, and this ability rests primarily on the uninterrupted nature of the input. Here we explore the impact of altering how visual information is accumulated over time by assessing how intermittent vision influences memory retention. Previous work has shown that intermittent, or stroboscopic, visual training (i.e., practicing while only experiencing snapshots of vision) can enhance visual-motor control and visual cognition, yet many questions remain unanswered about the mechanisms that are altered. In the present study, we used a partial-report memory paradigm to assess the possible changes in visual memory following training under stroboscopic conditions. In Experiment 1, the memory task was completed before and immediately after a training phase, wherein participants engaged in physical activities (e.g., playing catch) while wearing either specialized stroboscopic eyewear or
\end{abstract}

Electronic supplementary material The online version of this article (doi:10.3758/s13414-012-0344-6) contains supplementary material, which is available to authorized users.

L. G. Appelbaum $(\bowtie) \cdot$ M. S. Cain • E. F. Darling $\cdot$ S. R. Mitroff

Center for Cognitive Neuroscience, Duke University,

Box 90999, Durham, NC 27708-0999, USA

e-mail: greg@duke.edu

L. G. Appelbaum

Department of Psychiatry and Behavioral Sciences,

Duke University,

Durham, NC, USA

M. S. Cain • E. F. Darling $\cdot$ S. R. Mitroff

Department of Psychology and Neuroscience, Duke University, Durham, NC, USA

J. E. Schroeder

Department of Psychology, University of California,

Los Angeles, CA, USA transparent control eyewear. In Experiment 2, an additional group of participants underwent the same stroboscopic protocol but were delayed $24 \mathrm{~h}$ between training and assessment, so as to measure retention. In comparison to the control group, both stroboscopic groups (immediate and delayed retest) revealed enhanced retention of information in short-term memory, leading to better recall at longer stimulus-to-cue delays $(640-2,560 \mathrm{~ms})$. These results demonstrate that training under stroboscopic conditions has the capacity to enhance some aspects of visual memory, that these faculties generalize beyond the specific tasks that were trained, and that trained improvements can be maintained for at least a day.

Keywords Plasticity · Visual memory · Sensory memory · Visual short-term memory - Stroboscopic vision · Generalized learning $\cdot$ Attention in learning $\cdot$ Visual perception

Exposing an organism to an altered visual environment often results in modifications to the visual system of the organism (e.g., Blake \& Hirsch, 1975; Webster, Mizokami, $\&$ Webster, 2007). As humans, our typical visual experiences begin with a continuous and uninterrupted flow of incoming information, and our visual system has developed the ability to transform this information into a seamless and continuous perceptual experience of motion and form. What would happen if the stream of input to the visual system were altered to only allow successive glimpses of the world?

Intermittent, or stroboscopic, vision provides an interesting manipulation because it interrupts the normal flow of visual information, and therefore reduces the feedback that is available to guide movements as they are carried out. For example, imagine trying to catch a ball while in a stroboscopic environment. Because you cannot see continuously, you are forced to extrapolate between discrete visual 
samples to correctly judge the ball's trajectory. If the stroboscopic rate is sufficiently fast, visual feedback is frequent, and catching the ball is not complicated. However, as the rate decreases, more time passes between visual samples, and essential visual information is lost. Under these conditions, trying to catch a thrown ball becomes quite difficult.

When placed in a stroboscopic environment, one must adjust to perform visual tasks adequately, and several possible mechanistic changes could be made. For example, individuals may seek to compensate for their lack of continuous vision by preferentially storing information in visual memory to facilitate perception and/or motor control. This need for compensation suggests that stroboscopic environments may offer an especially powerful research tool for studying visual processing and the interplay between vision and motor actions.

Previous research has used intermittent visual experience to study what aspects of sight are important for regulating perceptual-motor performance, such as during driving (Senders et al., 1967), one-handed catching (Lyons, Fontaine, \& Elliot, 1997), or manual aiming (Elliott, Chua, $\&$ Pollock, 1994). More recently, training in a stroboscopic environment was shown to enhance visual sensitivity in a number of "low-level" perceptual domains, including foveal motion sensitivity and transient attentional selectivity (Appelbaum et al., 2011). For example, participants who practiced physical activities under stroboscopic conditions were more accurate afterward at reporting briefly presented stimuli in a dual-task setting than were matched controls who performed the same physical activities under full-vision conditions. Similarly, improvements in anticipatory timing have been observed after stroboscopic training (Smith \& Mitroff, under review), and professional ice hockey players have been found to improve in their on-ice skills after stroboscopic training (Mitroff et al., unpublished manuscript). While not all aspects of visual cognition tested in these studies were altered by stroboscopic training (suggesting that the observed effects were not solely due to motivational differences; see the Discussion section), the findings above indicate that experience with stroboscopic vision can enhance some aspects of perception and visual-motor control.

In the present study, we expanded upon these research findings to investigate the hypothesis that stroboscopic vision may force individuals to more robustly engage visual memory for successful motor planning (e.g., through greater retention of motion samples in order to estimate motion trajectories), and that this increased engagement may lead to enhancements in the early stages of visual memory. In particular, we focused here on the interplay between visual sensory memory and visual short-term memory. Visual sensory memory, also called "iconic memory" (Neisser, 1967), refers to the very brief, precategorical, representation of a visual stimulus that persists following the disappearance of that item (Coltheart, 1980; Gegenfurtner \& Sperling, 1993; Sperling, 1960). This initial, short-lived, and highcapacity system is accessible for several hundred milliseconds and has been shown to be a critical component of motor planning (Elliott et al., 1990, 1994). In the second stage of memory, "visual short-term memory," a subset of the elements contained in the initial sensory buffer are amplified and sustained for a short period, thereby creating a more durable memory store (Chun \& Potter, 1995; Hoffman, 1979). This second stage has been shown to depend on top-down factors, such as attention (e.g., Awh, Vogel, \& Oh, 2006; Cowan \& Morey, 2006), and to be susceptible to training-based plasticity and learning (Berry et al., 2010; Klingberg, 2010; Westerberg et al., 2007).

To assess possible effects on visual memory due to stroboscopic experiences, participants in the present study either completed an experimental condition, in which they trained with stroboscopic eyewear, or a control condition, in which they underwent identical training with nonstroboscopic eyewear. Visual memory was then assessed either immediately after training (Exp. 1) or after a 24-h delay (Exp. 2), which provided a means to test for both immediate and delayed changes to performance as a result of stroboscopic training. Stroboscopic vision was created via Nike Vapor Strobe ${ }^{\mathrm{TM}}$ eyewear, which contains liquid-crystal-filtered plastic lenses that can alternate between clear and opaque states. The clear state is held constant at $100 \mathrm{~ms}$ and the opaque state (which is a dark gray) can vary along eight levels between 67 and $900 \mathrm{~ms}$. The nonstroboscopic eyewear was identical except that the lenses were replaced with clear plastic. The training consisted of multiple sessions of athletic activities during which participants performed simple drills such as throwing and catching.

All participants were tested on a modified "partial-report task" (Lu et al., 2005; Sperling, 1960) before and after training. This task measures memory retention by cueing participants at various delays to report the identity of a subset of items from a larger display that was briefly presented and removed. Because participants do not know which items will be cued for recall, performance on this task can be regarded as a random sample of the memory for the entire display, thereby providing a means to assess memory retention at delays spanning sensory and short-term durations (Long, 1980). Alterations in the time course and capacity of memory were assessed through psychometric modeling of three parameters (see $\mathrm{Lu}$ et al., 2005): the initial visual availability of stimulus information $\left(a_{1}\right)$; the duration of sensory memory, determining how much information is available for transfer into short-term memory $(\tau)$; and the amount of information retained in short-term memory $\left(a_{0}\right)$. 


\section{Experiment 1: Immediate assessment}

Method

\section{Participants}

For Experiment 1, 84 participants completed the full training and testing protocol, and they were drawn from two cohorts: in-lab and team-based. In-lab participants were members of the Duke University community and were recruited through campus advertisements and the Psychology Department participant pool. Team-based participants were members of three Duke University varsity athletic teams (men's soccer, women's soccer, and men's basketball) and were recruited through the team coaches and athletic trainers. For all analyses, we categorized participants into one of two training cohorts: ${ }^{1}$ in-lab participants or varsity athletes (there were no differences across the three participating teams, so we collapsed their data). Participants were advised not to participate if they had a history of seizures, migraines, or light sensitivity. Each participant was compensated for the computer-based testing with either cash or with experiment participation credit in partial fulfillment of a Psychology Department requirement. Voluntary informed consent was obtained for every session in accordance with the Duke University Institutional Review Board.

Of the 84 participants, 43 were assigned to the strobe condition and 41 to the control. One participant from each group was excluded for failure to follow directions. The data were then prescreened for outliers whose overall accuracy on the partial-report task fell \pm 2 standard deviations from the group average. On the basis of these criteria, the data from two strobe and three control participants were removed, leaving 40 strobe and 37 control participants in the final analysis.

\section{Study design}

Each participant completed two aspects of the study: computer-based assessments and visual-motor training. The computer-based assessments were administered prior to training and immediately after the final training session. Participants were assigned to either the strobe training condition or the control training condition randomly, for the inlab participants, and pseudorandomly by athletic skill set, for the team-based participants (e.g., half of the soccer forwards were randomly assigned to the strobe condition and half to the control condition).

\footnotetext{
${ }^{1}$ For clarity, we use the term "cohort" to refer to the different collections of training participants (in-lab vs. varsity athletes) and "condition" to refer to the strobe versus control training regimens.
}

\section{Computer-based iconic memory assessment}

Computer-based assessments were administered via Dell Inspiron computers running MATLAB R2010a and the Psychophysics Toolbox (http://psychtoolbox.org). All computers were attached to CRT monitors that were calibrated in order to assure that all visual stimuli were the same size, regardless of slight differences in screen size. Monitors were set to a $75-\mathrm{Hz}$ screen refresh and 1,280 $\times 1,024$ resolution. Assessments for the in-lab participants were collected in the Visual Cognition Laboratory at Duke University with one or two participants at a time. Team-based participants either completed the assessments in the same lab or in a temporary computer lab created in the basketball practice facility, which could accommodate up to four participants at the same time. Some team-based participants also performed additional computer-based assessments measuring motion interpolation (flash-lag effect) and simple reaction times, but these tasks were not included in the final analysis because of the more limited sample size.

The partial-report task was a modified version of the task from Lu et al. (2005). Visual stimuli (Fig. 1) were viewed by the participants from an approximate distance of $57 \mathrm{~cm}$ in a dimly lit room. Each trial began with a black fixation cross at the center of a gray $\left(30 \mathrm{~cd} / \mathrm{m}^{2}\right)$ background. After $400 \mathrm{~ms}$, eight black uppercase letters (each $1.3^{\circ} \times 1.3^{\circ}$ ) appeared for $105 \mathrm{~ms}$, arranged on an imaginary circle $\left(3.50^{\circ}\right.$ radius $)$ around fixation. The letters were drawn randomly from the set "D," "F," "J," and "K," with the constraint that no neighboring letters were the same. The letter display was replaced by a fixation cross and then a red line $\left(1^{\circ}\right.$ in length with a circle at the end) appeared after a variable delay, or interstimulus interval (ISI), of 13, 40, 80, 160, 320, 640, 1,280 , or $2,560 \mathrm{~ms}$. The line pointed randomly at one of the eight letter locations and remained visible until a response was given. The participants were to report the identity of the letter at the cued location using the corresponding key on a standard keyboard. To provide a performance baseline with no memory component, some previous partial-report studies had implemented precue and simultaneous-cue conditions (e.g., in $\mathrm{Lu}$ et al., 2005, where the arrow could also appear $147 \mathrm{~ms}$ before or simultaneously with the letter array). These conditions were omitted here for logistical reasons, as we wanted to present a difficult task and were under a time constraint for some testing sessions. Importantly, these baselines were not necessary for the present purposes, since we were comparing performance within individuals from their pretraining to their posttraining sessions.

\section{Stroboscopic training regimens}

The activities engaged in during the stroboscopic training were tailored to each participant cohort, but importantly, the 
Fig. 1 Depiction of a sample trial of the partial-report task (not drawn to scale). Each trial started with a central fixation that was replaced by a circular array of eight letters. Participants were instructed to maintain central fixation, and then after a variable delay a red line (shown here in black) appeared at fixation indicating the location of the target letter, which the participant was to report via a keyboard buttonpress

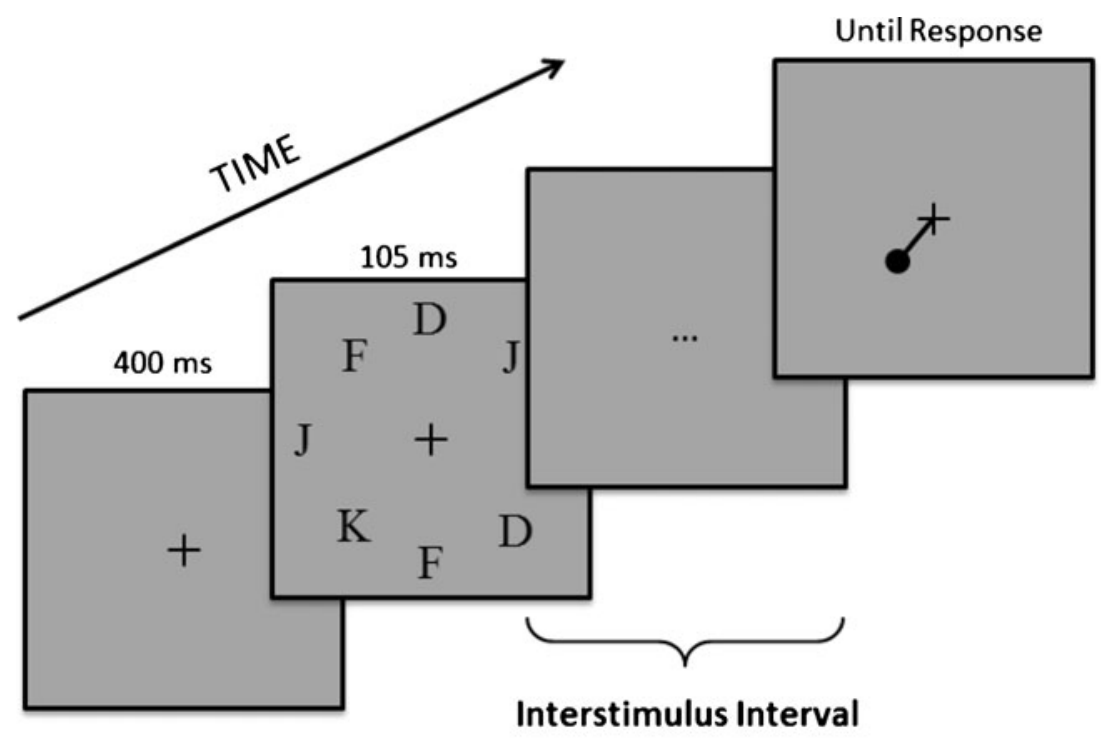

$13,40,80,160,320,640,1280$ or $2560 \mathrm{~ms}$ strobe condition and the control condition were always run in the same manner within each cohort. Prior to training, participants were instructed how to operate the eyewear. They trained with the eyewear for a specified duration in each training session, as is described for the three training cohorts below and summarized in Table 1. In general, training began at the fastest (i.e., easiest) strobe rate $(6 \mathrm{~Hz})$ and was made progressively harder by reducing the strobe rate (i.e., increasing the occlusion length) over the course of the training. (Note: The visible period was always constrained to be $100 \mathrm{~ms}$.)

In-lab: participants, training, and assessments A group of 58 participants made two visits to the lab and undertook the same training regimen reported in Appelbaum et al. (2011). On the first day, individuals completed the computer-based assessments and then participated in a 27-min training session that consisted of forward-facing and turn-and-catch drills (see Appelbaum et al., 2011, for more details). For the strobe group participants, the strobe eyewear started at Level 1 and was increased up to Level 6 on the basis of catching performance (control participants underwent the same procedure, but the

Table 1 Summary of Experiment 1 training cohorts

\begin{tabular}{|c|c|c|c|c|}
\hline Cohort & $\begin{array}{l}\text { \# of } \\
\text { Sessions }\end{array}$ & $\begin{array}{l}\text { Session } \\
\text { Length }\end{array}$ & Activities & $\begin{array}{l}\text { \# of } \\
\text { Participants }\end{array}$ \\
\hline $\begin{array}{l}\text { In-lab } \\
\text { training }\end{array}$ & 2 & $27 \mathrm{~min}$ & Catch & 58 \\
\hline $\begin{array}{l}\text { Varsity } \\
\text { soccer }\end{array}$ & 6 or 7 & $15-45 \mathrm{~min}$ & $\begin{array}{l}\text { Agility and ball } \\
\text { handling drills }\end{array}$ & 16 \\
\hline $\begin{array}{l}\text { Varsity } \\
\text { basketball }\end{array}$ & 5 or 6 & $15-40 \mathrm{~min}$ & $\begin{array}{l}\text { Agility and ball } \\
\text { handling drills }\end{array}$ & 10 \\
\hline
\end{tabular}

eyewear remained transparent throughout). All participants returned to the lab within $48 \mathrm{~h}$ to complete a second, identical training session and then were immediately readministered the iconic memory computer-based assessments. In-lab training was conducted by members of the research team in a well-lit 20-foot hallway near the computer assessment room.

Men's and women's varsity soccer: participants, training, and assessments Members of the men's $(n=8)$ and women's $(n=8)$ soccer teams participated in a multiday training version of this study. Computer assessments were administered in the Visual Cognition Laboratory within one week prior to the start of the first training session. The men's team completed six training sessions, and the women's team completed seven. All sessions, except for the final one, were conducted at the teams' practices and consisted of typical soccer activities, such as passing and dribbling drills. Participants on each team were split evenly into the strobe and control conditions. The stroboscopic frequency level for the strobe condition varied across training sessions-during some drills, training was done at a single rate, and for others the frequency was altered from faster to slower rates at set time intervals between organized drills. Overall, strobe condition participants primarily experienced Levels $2-4$ (5$3 \mathrm{~Hz}$ ). The control participants did everything exactly the same, including pressing the buttons on the eyewear to change the level, but their lenses remained transparent throughout. The final training session for each team member was conducted outside of the Visual Cognition Laboratory (in participant pairs or with a lab member). This session lasted $24 \mathrm{~min}$, was modeled after the teams' practice sessions, and was completed immediately before the posttraining computer-based assessments. The time from the initial training session to the final 
training session and posttraining assessment was no more than two weeks for any participant. Physical measures (cone dribbling times) were also collected, but are not reported here.

Men's varsity basketball: participants, training, and assessments Computer-based assessments were administered before and after training. All testing and training were conducted in the basketball training building on campus. Ten team members (six strobe/four control) participated in five or six total training sessions that were led by coaches, athletic trainers, or senior members of the team. These sessions were completed within an eight-day period. The training consisted of warm-up and agility drills, with variability in timing (between 15 and $40 \mathrm{~min}$ ) and activities across sessions. The stroboscopic frequency level for the basketball team varied across training sessions, with participants primarily experiencing Levels $2-4(5-3 \mathrm{~Hz})$, and control participants mimicking the same procedure but with eyewear that remained transparent throughout. The final training session took place immediately before the posttraining computer-based assessments. A physical measure (freethrow shooting) was collected, but is not reported here.

\section{Results}

All participants produced typical memory decay functions, with high accuracy at short ISIs and performance falling to near chance at the longest ISI (Fig. 2a). At the pretraining assessment, performance did not differ between the strobe and control conditions at any ISI (all $p$ values $>.18$ ). For both training conditions, performance improved from pretraining to posttraining; however, the magnitude of this improvement was significantly larger in the strobe participants than the control participants (Fig. 2b). A mixed-model ANOVA

\section{a) Partial Report Performance}

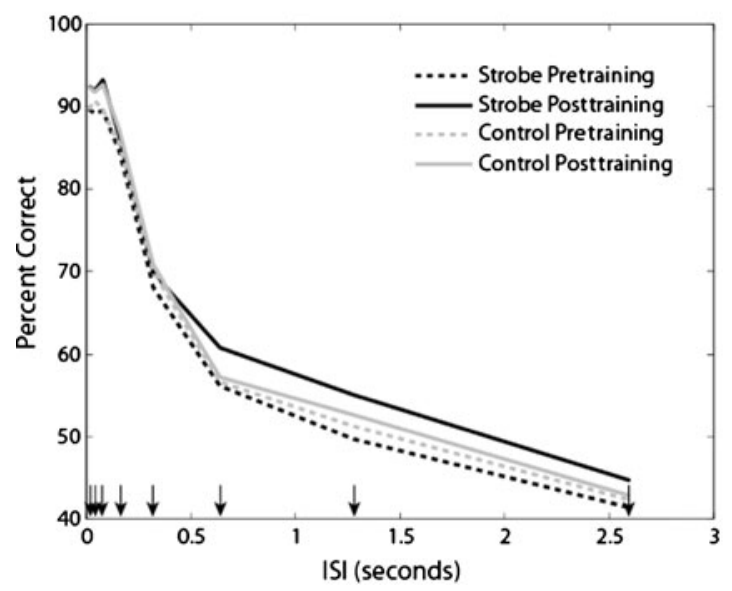

Fig. 2 a Accuracy as a function of interstimulus interval (ISI) for the strobe (black) and control (gray) measures at pretraining (dashed lines) and at posttraining (solid lines). b Retest improvement for the strobe performed on the accuracy data with the betweenparticipants factors Cohort (in-lab vs. varsity) and Condition (strobe vs. control) and the within-participants factors Session (pre vs. post) and ISI (eight delays) revealed significant within-participants main effects of Session $[F(1,73)=$ $20.34, p<.001]$ and ISI $[F(7,289.34)=670.00$, $p<.001]$, and a significant Session $\times$ Condition interaction $[F(1,73)=4.95, p=.029]$. The between-participants factor Cohort was not significant $[F(1,73)=0.66, p=.42]$ and did not interact with any other factors. This lack of a cohort effect suggests that none of the potential differences between the inlab and team-based participants (e.g., number of training sessions, initial athletic skill, knowledge of more than one experimental condition, or location of training) had a meaningful impact.

Paired comparisons of the pre- to posttraining differences (Table 2) for the two conditions indicated that while both training regimens resulted in improved performance for several of the short ISIs, only the strobe training resulted in significantly improved performance at the longer ISIs $(\geq 640 \mathrm{~ms})$

\section{Parameter estimates}

In order to characterize the temporal properties of iconic memory, the data were converted from four-alternative forced identification to $d^{\prime}$ (a measure of sensitivity; see, e.g., Wickens, 2001), and the following exponential-decay function was fit to the data:

$d^{\prime}(\mathrm{ISI})=a_{0}+a_{1} e^{-\mathrm{ISI} / \tau}$.

In this three-parameter function, $a_{1}$ is the fast-decaying sensitivity that reflects the initial visual availability of stimulus information, $\tau$ is the time constant of the fast-decaying

\section{b) Retest Improvement}

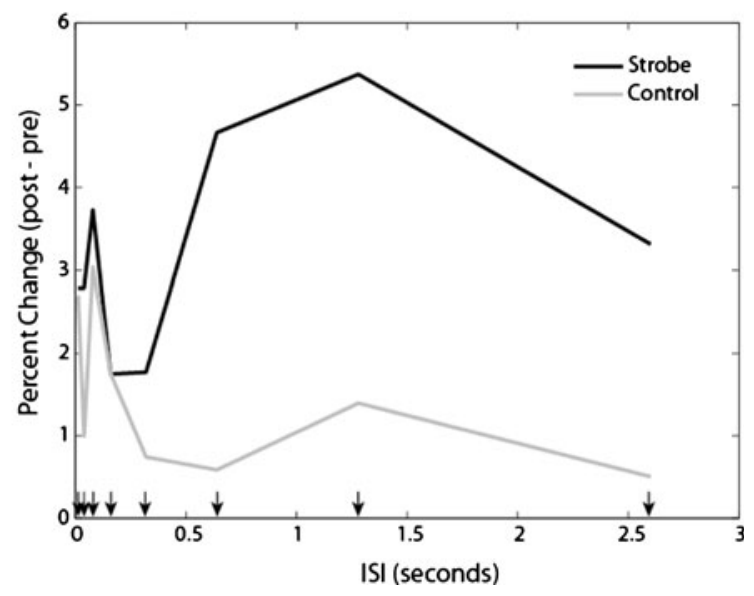

(black) and control (gray) groups. The ISIs tested in the present experiment are indicated by arrows at the bottom of each plot 
Table 2 The $p$ values for paired comparisons between pretraining and posttraining accuracy at each ISI

\begin{tabular}{lll}
\hline ISI (ms) & Strobe & Control \\
\hline 13 & $t(39)=2.45, p=.010^{*}$ & $t(36)=1.97, p=.028^{*}$ \\
40 & $t(39)=2.69, p=.005^{*}$ & $t(36)=0.98, p=.166$ \\
80 & $t(39)=2.83, p=.004^{*}$ & $t(36)=2.30, p=.014^{*}$ \\
160 & $t(39)=1.15, p=.128$ & $t(36)=1.11, p=.137$ \\
320 & $t(39)=0.82, p=.209$ & $t(36)=0.57, p=.287$ \\
640 & $t(39)=3.67, p<.001^{*}$ & $t(36)=0.31, p=.379$ \\
1,280 & $t(39)=2.70, p=.005^{*}$ & $t(36)=0.71, p=.240$ \\
2,560 & $t(39)=1.83, p=.038^{*}$ & $t(36)=0.28, p=.391$ \\
\hline
\end{tabular}

${ }^{*} p<.05$ (one-tailed)

sensitivity that represents the duration of iconic memory, and $a_{0}$ is the sensitivity at long delays that reflects the amount of information transferred into short-term memory without the benefit of cuing (see Lu et al., 2005). The corresponding model parameters were derived for each participant's pretraining and posttraining performance (Table 3A).

Paired comparisons (one-tailed) between the parameter estimates (see Table $3 \mathrm{~A}$ and $\mathrm{B}$ ) revealed a significant increase in the $a_{1}$ parameters for both the strobe $(p=.006)$ and control $(p=.04)$ conditions, indicating that the fastdecaying sensitivity to the initial visual stimulus information was improved at retest for both conditions. This retest improvement was statistically equivalent for the two groups $(p=.371)$. No difference was observed for either group on the $\tau$ parameter (and there was not a significant difference between conditions, $p=.299$ ), indicating that the time constant of the fast-decaying sensitivity that represents the duration of sensory memory was not changed at retest.

Table 3 (A) Best-fitting parameters for the strobe and control conditions at pretraining and posttraining, as well as the pairedcomparisons significance levels of their differences. (B) Posttraining
Confirming the differences between conditions in retest improvement at the longer ISIs shown in Fig. 2, stroboscopic training resulted in a significantly higher $a_{0}$ parameter estimate at retest $(p=.019)$, while control training did not produce a statistically significant difference in this parameter $(p=.303)$. The significant difference between conditions on retest improvement in this parameter $(p=.035)$ indicates that stroboscopic training increased the amount of information retained in short-term memory.

\section{Experiment 2: 24 hour delayed assessment}

The goal of this experiment was to examine whether the training effects observed in Experiment 1 for the strobe group would last for $24 \mathrm{~h}$ after the final training session. Such retention is an important facet of learning, and here we probed this issue by testing a separate group of participants with an approximately 24-h delay inserted between training and the posttraining assessment. We chose this time frame because it involves an additional night of sleep, and sleepdependent memory consolidation is known to improve learning (e.g., Censor, Karni, \& Sagi, 2006; Mednick et al., 2009).

\section{Method}

All aspects of this experiment were identical to those of Experiment 1, except for the following points. The participants $(n=33)$ were all run through the in-lab training protocol, and all completed training using the strobe eyewear. Two assessments were collected on each participant; the modified partial-report task described in Experiment 1, and a motion coherence task (see Appelbaum et al., 2011).

minus pretraining differences in parameter estimates with the betweensubjects comparisons significance levels

$\begin{array}{lll}a_{1} & a_{0}\end{array}$

(A) Parameter estimates

$\begin{array}{lllll}\text { Strobe } & \text { Pretraining } & 2.903 & 0.474 & 0.545 \\ & \text { Posttraining } & 3.318 & 0.473 & 0.683 \\ & \text { Paired } t \text { test } & t(39)=2.62, p=.006^{*} & t(39)=-0.02, p=.495 & t(39)=2.14, p=.019^{*} \\ \text { Control } & \text { Pretraining } & 3.041 & 0.442 & 0.614 \\ & \text { Posttraining } & 3.356 & 0.496 & 0.578 \\ & \text { Paired } t \text { test } & t(36)=1.79, p=.04^{*} & t(36)=0.81, p=.210 & t(36)=-0.52, p=.303 \\ \text { (B) Posttraining } & \text { minus pretraining difference in parameter estimates } & & 0.137 \\ & \text { Strobe } & 0.415 & -0.001 & -0.036 \\ & \text { Control } & 0.314 & 0.054 & t(75)=1.84, p=.035^{*} \\ & \text { Between-subjects } t \text { test } & t(75)=0.31, p=.335 & t(75)=0.53, p=.299 & \end{array}$

$*_{p}<.05$ (one-tailed) 
These assessments were counterbalanced in order, and we will focus here on the memory task (the motion coherence data can be found in the supplementary materials). Participants were recruited for a three-day protocol: Day 1, pretraining assessments and first in-lab training session; Day 2 , second in-lab training session; Day 3, posttraining assessments. The posttraining assessment was administered the day following the second and final training session and was scheduled to be as close to $24 \mathrm{~h}$ later as possible, given the participants' scheduling constraints (mean $=23 \mathrm{~h} 5 \mathrm{~min}$, standard deviation $=1.99 \mathrm{~h}$ ). The data from two participants were excluded from the final analysis, as their mean accuracy was greater than two standard deviations from the group's mean.

By maintaining all other aspects of the strobe condition of Experiment 1, this "retention" condition provided a direct means to explore preservation of the stroboscopic training effects: We could simply compare the parameter estimates produced here to those from the strobe and control conditions from Experiment 1. If there was significant retention of the stroboscopic training, the parameters should be significantly different from those in Experiment 1's control condition, but not from those in the strobe condition.

\section{Results}

\section{Retention condition}

As was observed in Experiment 1, the retention condition produced typical memory decay functions (Fig. 3a) and demonstrated improvement from pretraining (dashed) to posttraining (solid). An ANOVA performed on the accuracy data for the within-participants factors

a) Partial Report Performance

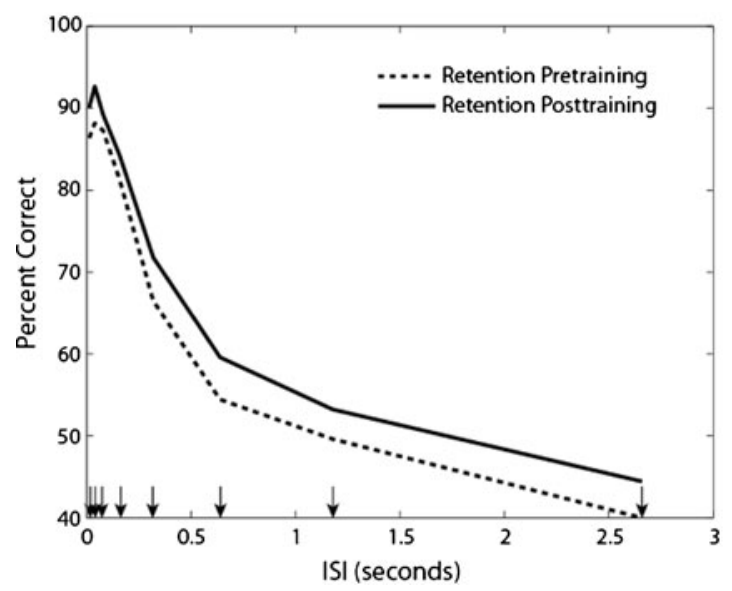

Fig. 3 a Accuracy as a function of interstimulus interval (ISI) for the retention condition (Exp. 2) at pretraining (dashed line) and at posttraining (solid line). b Retest improvement for all three conditions
Session (pre vs. post) and ISI (eight delays) revealed significant main effects of Session $[F(1,30)=25.36, p<.001]$ and ISI $[F(3.54,106.2)=299.27, p<.001]$, as well as a nonsignificant interaction $[F(4.66,139.9)=0.475, p=.78]$. Paired comparisons of the pre- to posttraining differences, shown in Table 4, indicated that the main effect of retest was driven by significant improvement at a number of the short and long ISIs (gray shading).

Comparison between the strobe, control (Exp. 1), and retention (Exp. 2) conditions

The primary goal of Experiment 2 was to examine whether the stroboscopic training effects observed in Experiment 1 could be retained over a 24-h delay. A significant main effect of session and significant paired-comparison $t$ tests (cf. Table 3) demonstrated that the retention condition did, in fact, result in improved visual sensory memory performance. However, as can be seen in Fig. 3a, there were some differences between the retention condition and the strobe and control conditions from Experiment 1. For example, the retention condition produced a significant difference from pretraining to posttraining for the 320 -ms ISI (Table 4), whereas neither the strobe nor the control conditions did so in Experiment 1 (Table 2).

The parameter estimates $a_{1}, \tau$, and $a_{0}$ offer the most direct means to compare the retention condition to the strobe and control conditions. Estimates were derived for 30 of the 31 retention participants, with one excluded participant having estimates that failed to converge. For the retention condition, paired-comparison one-tailed $t$ tests for the pretraining and posttraining estimates revealed significant increases for $a_{1}$ $[t(29)=2.43, p=.011]$ and $a_{0}[t(29)=1.88, p=.035]$, but not for $\tau[t(29)=0.81, p=.22]$ (white bars in Fig. 4).

\section{b) Retest Improvement}

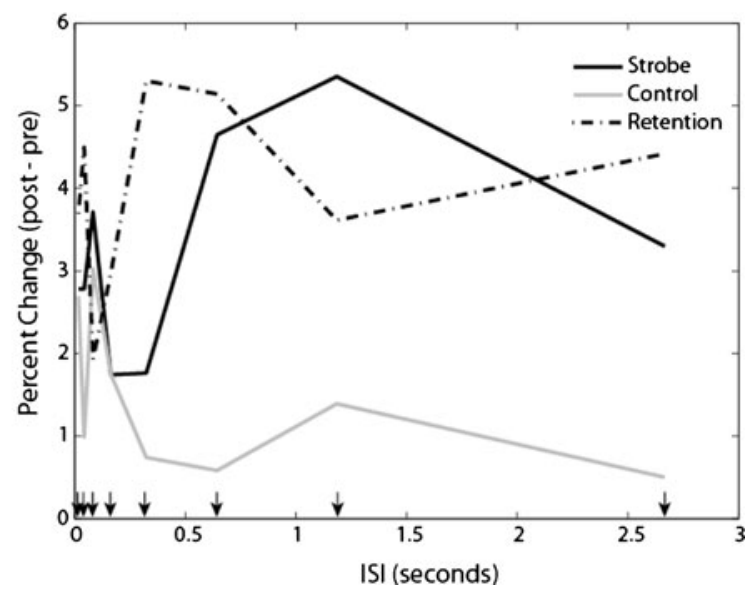

(Exps. 1 and 2) across ISIs. The ISIs tested are indicated by arrows at the bottom of each plot 
Table 4 The $p$ values for paired comparisons between pretraining and posttraining accuracy at each ISI for the retention condition

\begin{tabular}{ll}
\hline ISI (ms) & Retention Condition \\
\hline 13 & $t(30)=2.19, p=.018^{*}$ \\
40 & $t(30)=3.65, p<.001^{*}$ \\
80 & $t(30)=1.30, p=.101$ \\
160 & $t(30)=1.67, p=.052$ \\
320 & $t(30)=2.95, p=.003^{*}$ \\
640 & $t(30)=2.15, p<.020^{*}$ \\
1,280 & $t(30)=1.59, p=.061$ \\
2,560 & $t(30)=1.93, p=.031^{*}$ \\
\hline
\end{tabular}

Asterisk $p<.05$ (one-tailed)

To compare the retention condition to the Experiment 1 conditions, a separate one-way ANOVA was conducted for each parameter estimate (see Fig. 4). The $a_{1}$ parameter did not differ across the three conditions $[F(2,106)=0.37, p=$ .69], indicating that comparable improvements were seen for all three groups. The $\tau$ parameter, which was not reliably changed from pre- to posttraining for any of the three conditions, also did not differ significantly across conditions $[F(2$, 106) $=0.54, p=.58]$. The $a_{0}$ parameter revealed a trending relationship across the three conditions $[F(2,106)=2.26, p=$ .11]. Having already established a difference between the strobe and control conditions in Experiment 1, planned paired comparisons were conducted between each of these Experiment 1 conditions and the retention condition. These comparisons demonstrated that there was no significant difference between the retention and strobe conditions $[t(68)=0.33$, $p=.37]$, but there was between the retention and control conditions $[t(65)=1.84, p=.035]$, thereby supporting the view that training-based improvements were maintained for at least $24 \mathrm{~h}$.

\section{Discussion}

Previous research has shown that stroboscopic training can improve performance, but much remains unknown about how it can improve performance. This important question has begun to be answered with evidence that stroboscopic training can affect processes such as anticipatory timing (Smith \& Mitroff, under review), eye-hand coordination (Bennett et al., 2004; Mitroff et al., unpublished manuscript), and basic perceptual abilities (Appelbaum et al., 2011). The goals of the present study were to determine whether measurable changes to visual memory could also be observed, how this would manifest across the different stages of visual memory, and whether such improvement could be retained for a day following the training.

The results from Experiment 1 demonstrated an increase in short-term memory capacity following stroboscopic training. Parameter estimates of the time course of visual memory informed us about what aspects of the process were affected by the training. First, both the strobe and control conditions experienced significant increases in initial visual sensitivity at retest (parameter $a_{1}$ ), suggesting a general testretest improvement in the ability to process the incoming visual information. Second, neither group experienced a change in the duration of sensory memory (parameter $\tau$ ), suggesting no effect of training on the ability to transfer information from sensory memory into short-term memory. Third, and critically, only the strobe group experienced improved performance at long cue delays (parameter $a_{0}$ ), indicating a specific benefit of stroboscopic training that led

\section{Parameter Estimate Differences (posttraining minus pretraining)}

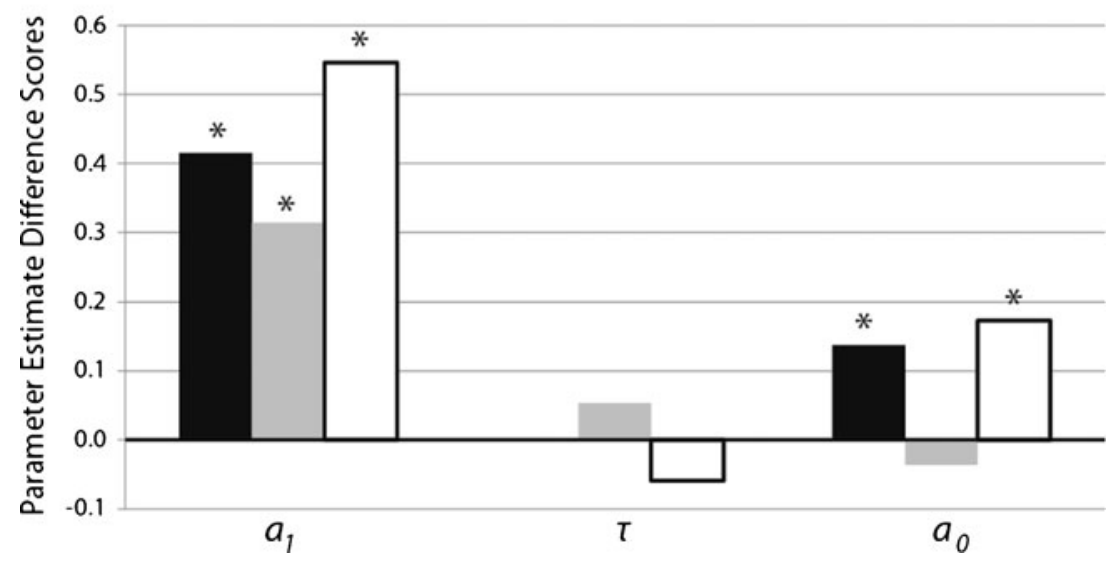

Fig. 4 Posttraining minus pretraining parameter estimates for the strobe, control, and retention conditions (black, gray, and white bars, respectively). A significant improvement in $a_{1}$ emerged for all three conditions, and no significant training effect on $\tau$ for any condition.
The strobe and retention conditions revealed significant improvements for $a_{0}$, but the control condition did not, indicating improvements in visual memory encoding from stroboscopic training that was retained for at least $24 \mathrm{~h}$ following training. ${ }^{*} p<.05$ 
to an increase in the amount information that could be retained in the more durable short-term memory store. The results of Experiment 2 replicated the strobe condition findings from Experiment 1 with a posttraining assessment coming after a $24 \mathrm{~h}$ delay. This indicates that the observed visual memory benefits were not only immediate, but were maintained after training.

\section{Implications for the study of visual memory}

Visual memory involves the ability to store and retrieve previously experienced visual sensations when the original stimuli are no longer present. This ability is typically conceptualized as reflecting three separate temporal stages; sensory memory, short-term memory, and long-term memory (Atkinson \& Shiffrin, 1968). Residing at the front end of this memory cascade, sensory memory is a crucial faculty that allows some characteristics of our sensory experience to be preserved very briefly in a high-capacity, precategorical buffer after they have already disappeared from sight (Coltheart, 1980; Di Lollo, 1977; Long, 1980; Sperling, 1960). With very short presentations, individuals often report that they seem to "see" more than they can actually report, thereby supporting the notion that information that is not quickly transferred into a durable memory store is lost and inaccessible for later recollection.

This transfer and retention of information through the early stages of memory has typically been explored using cued partial-report tasks, such as the one employed in the present study. In these tasks, an interval is introduced between a stimulus array and the presentation of a cue, which prompts the participant to report the contents of memory at a given location. When the cue is presented, participants are able to focus attention on the cued content and begin transferring that information into the more durable short-term memory storage, a process that Gegenfurtner and Sperling (1993) called "selective transfer." While such cued-retrieval strategies allow individuals to preferentially encode some information into short-term memory, it is also clearly the case that not all memory is the result of selective transfer. For example, at very long stimulus-to-cue delays, partialreport accuracy does not decay all the way to zero, indicating that "nonselective transfer" occurs in the absence of any explicit cue (Averbach \& Coriell, 1961).

In the present study, we tested for the effects of stroboscopic visual training on visual memory. The results indicated two forms of learning that may differentially influence selective and nonselective visual memory transfer. The most relevant for the present question is that stroboscopic training increased the overall number of items encoded into shortterm memory at long stimulus-to-cue delays, a form of nonselective transfer. As reflected by the significant session-by-condition interaction, subsequent paired tests, and changes in the $a_{0}$ model parameter estimates, training under stroboscopic conditions resulted in greater retest performance at the longer ISIs, whereas no such improvement was seen for the control participants. This finding indicates that more information is being registered into short-term memory, without the benefit of cuing, as a result of stroboscopic experience. Interestingly, this improvement in nonselective transfer was retained over a 24 -h interval in the retention condition, indicating a relatively stable form of learning.

The second form of learning revealed in the present study is that individuals in all three conditions (strobe, control, and retention) showed significant retest improvements at short ISIs (and, similarly, in the $a_{1}$ model parameter estimates). This finding suggests that at retest all three groups experienced a significant increase in their initial visual sensitivity and selective transfer of information into memory occurring with short stimulus-to-cue intervals. The lack of any differential effect due to the training conditions, however, indicates that this stage of memory was not specifically affected by stroboscopic exposure, or an additional 24-h delay before the retest, but rather was generally enhanced, likely due to practice with the partialreport task.

Somewhat unexpectedly, the time constant of sensory memory decay $(\tau)$ did not differ at retest for any of the groups. It has been suggested that the duration of sensory memory, as captured by this decay parameter, reflects the rate at which individuals are able to switch attention between elements maintained in memory (Gegenfurtner \& Sperling, 1993). While it is difficult to make strong inferences from the lack of retest or group differences, the present findings suggest that sensory memory duration (and thus the ability to rapidly shift attention through the memory store) was not the limiting factor for memory capacity in this task. Rather, it appears from these data that improved short-term memory may be the cause of the observed improvements in performance.

In interpreting why stroboscopic training would selectively improve short-term memory retention, it is interesting to consider the nature of the training itself. For example, the in-lab participants played catch under stroboscopic conditions, which for all intents and purposes is a partial-report task. In this situation, the participants see a glimpse of the visual world with a ball about to be thrown or in flight, and then they are presented with an opaque visual environment, which is then again replaced by a glimpse of the visual world. They do their best to catch the ball, but there are multiple possible outcomes of where the ball could go and at what time it will arrive. As such, the better they are at retaining visual information in a usable memory store, the better they will be after the visual interruption imposed by the eyewear. 
Implications for the study of stroboscopic vision and training

Intermittent visual experience has been used to study which aspects of vision are important for regulating perceptualmotor performance (Elliott et al., 1994; Lyons et al., 1997; Senders et al., 1967). By manipulating visual input, researchers can test what information is important, when is it necessary, and for how long it must persist. Previous research has generally shown that although there are preferred sources of visual information, participants are able to adapt in degraded vision conditions by making optimal use of whatever sources of information the environment provides (Assaiante, Marchand, \& Amblard, 1989; Bennett et al., 2006; Bennett et al., 2004; reviewed in Elliott, 1990). Such studies have employed a wide variety of techniques and manipulations, including comparing how novice and expert athletes react to intermittent vision (e.g., Bennett et al., 2004; Robertson et al., 1994) and focusing on adaptation during intermittent vision (e.g., Lyons et al., 1997; Olivier et al., 1998). Likewise, previous studies have shown that shortlived visual memories can serve as a substitute for direct vision when the visible environment has been disturbed by a mask (Elliott et al., 1994).

The stroboscopic tool employed here, Nike Vapor Strobe ${ }^{\mathrm{TM}}$ eyewear, has been used previously to reveal effects on anticipatory timing (Smith \& Mitroff, under review), eye-hand coordination in professional athletes (Mitroff et al., unpublished manuscript), and various aspects of perceptual processing (Appelbaum et al., 2011). The present results add to this existing literature, suggesting that stroboscopic training can enhance visual memory abilities and that the effects can last for at least $24 \mathrm{~h}$ (see Smith \& Mitroff, under review, and Mitroff et al., unpublished manuscript, for further discussions of retention). This 24-h retention finding is important, given that training protocols are most significant when they produce lasting effects (Karni \& Sagi, 1993; Savion-Lemieux \& Penhune, 2005; Scott et al., 2008).

The findings above suggest that experience with stroboscopic vision can enhance aspects of perception and visualmotor control, yet it is also informative to know what stroboscopic training does not seem able to affect. To understand the mechanisms fostering these abilities, positive and negative effects can work in tandem to build a more complete story. For example, Appelbaum et al. (2011) found that stroboscopic visual training did not improve the ability to track multiple moving objects over several seconds. This suggests that stroboscopic training may not alter sustained attention abilities that do not involve the detection and processing of briefly presented information. Likewise, the same study found several occurrences of benefits for centrally presented visual information, but not for information in the periphery. More work is needed to further explore this result, but the effects of stroboscopic training for visual cognition abilities do not seem uniformly distributed across the visual field. Beyond constraining the possible underlying mechanism of stroboscopic training, these results also speak to concerns over placebo, motivational, or "Hawthorne" effects: The same participants who revealed significant effects in other paradigms run during the same testing session (and sometimes in the very same paradigm) did not reveal any significant differences on these measures. These findings help to alleviate concerns over such alternative accounts of stroboscopic training effects (see Appelbaum et al., 2011, for further treatment of this issue).

Moreover, while the eyewear employed here offers a convenient means to conduct training studies, as the stroboscopic environment is portable and can be restricted to some individuals and not others, it also presents a potential drawback: Portable stroboscopic eyewear proves extremely useful when conducting studies in a group setting, as the experimental and control groups can be simultaneously engaged in the same training, but this creates the possibility that the experimental and control groups may realize that there are two conditions and may be differentially motivated to perform (Boot, Blakely, \& Simons, 2011). Under this situation, might the experimental group be more motivated to try harder? While we cannot completely discount this possibility, the lack of cohort differences between our in-lab participants (who all completed the experiment one participant at a time and did not know about the various experimental conditions) and our varsity athlete participants suggests that motivation was not a critical concern. Moreover, previous work conducted in the same fashion had found several factors that diminish such concerns (see Appelbaum et al., 2011).

\section{Conclusion}

In the present study, we investigated stroboscopic vision to learn about the malleability of visual memory. After undergoing stroboscopic training, participants revealed an improved ability to retain visual information in short-term memory. Furthermore, this improved ability was still present $24 \mathrm{~h}$ later. While this is only one specific means by which visual processing can adapt, it indicates that stroboscopic training can lead to general improvements in higher-level visual cognition. More broadly, this result advances the scientific study of perceptual processing by providing an example of generalized learning. As well, this result informs athletic training by suggesting that stroboscopic experiences might be able to improve performance through benefits in visual memory. Sports often rely on the ability to keep fleeting information in memory (e.g., a basketball player making a no-look pass must remember the locations of his 
teammates and opponents), and any boost in visual memory abilities could manifest in improved performance.

Author note This research was funded by a grant to S.R.M. from Nike SST, Nike Inc. Thanks to the Duke athletic trainers (especially Jose Fonseca, Jeff Howser, Nick Potter, and Hap Zarzour) and coaches (especially Bryan Amos, Robbie Church, John Kerr, Billy Lesesne, and Mike Krzyzewski), and to all of the participants, as well as to Stephen Adamo, Christina Bogetich, Ben Crisp, Catherine Donatucci, Matt Forester, Sylvia Nantier, Elizabeth Price, Mike Schallmo, Ari Stern, and Gabby Villagomez. Thanks to Zhong-Lin Lu for providing the experimental program, and to Jeremy Wolfe and two anonymous reviewers for helpful feedback.

\section{References}

Appelbaum, L. G., Schroeder, J. E., Cain, M. S., \& Mitroff, S. R. (2011). Improved visual cognition through stroboscopic training. Frontiers in Psychology, 2, 276. doi:10.3389/fpsyg.2011.00276

Assaiante, C., Marchand, A. R., \& Amblard, B. (1989). Discrete visual samples may control locomotor equilibrium and foot positioning in man. Journal of Motor Behavior, 21, 72-91.

Atkinson, R. C., \& Shiffrin, R. M. (1968). Human memory: A proposed system and its control processes. In K. W. Spence \& J. T. Spence (Eds.), The psychology of learning and motivation (Vol. 2, pp. 89-195). New York, NY: Academic Press.

Averbach, E., \& Coriell, A. S. (1961). Short-term memory in vision. Bell Systems Technical Journal, 40, 309-328.

Awh, E., Vogel, E. K., \& Oh, S. H. (2006). Interactions between attention and working memory. Neuroscience, 139, 201-208. doi:10.1016/j.neuroscience.2005.08.023

Bennett, S., Ashford, D., Rioja, N., Coull, J., \& Elliott, D. (2006). Integration of intermittent visual samples over time and between the eyes. Journal of Motor Behavior, 38, 439-450.

Bennett, S., Ashford, D., Rioja, N., \& Elliott, D. (2004). Intermittent vision and one-handed catching: The effect of general and specific task experience. Journal of Motor Behavior, 36, 442-449.

Berry, A. S., Zanto, T. P., Clapp, W. C., Hardy, J. L., Delahunt, P. B., Mahncke, H. W., \& Gazzaley, A. (2010). The influence of perceptual training on working memory in older adults. PLoS ONE, 5.

Blake, R., \& Hirsch, H. V. (1975). Deficits in binocular depth perception in cats after alternating monocular deprivation. Science, 190, 1114-1116

Boot, W. R., Blakely, D. P., \& Simons, D. J. (2011). Do action video games improve perception and cognition? Frontiers in Psychology, 2, 1-6.

Censor, N., Karni, A., \& Sagi, D. (2006). A link between perceptual learning, adaptation and sleep. Vision Research, 46, 4071-4074. doi:10.1016/j.visres.2006.07.022

Chun, M. M., \& Potter, M. C. (1995). A two-stage model for multiple target detection in rapid serial visual presentation. Journal of Experimental Psychology. Human Perception and Performance, 21, 109-127. doi:10.1037/0096-1523.21.1.109

Coltheart, M. (1980). Iconic memory and visible persistence. Perception \& Psychophysics, 27, 183-228. doi:10.3758/BF03204258

Cowan, N., \& Morey, C. C. (2006). Visual working memory depends on attentional filtering. Trends in Cognitive Sciences, 10, 139-141. doi:10.1016/j.tics.2006.02.001

Di Lollo, V. (1977). Temporal characteristics of iconic memory. Nature, 267, 241-243. doi:10.1038/267241a0
Elliott, D. (1990). Intermittent visual pickup and goal directed movement: A review. Human Movement Science, 9, 531-548.

Elliott, D., Calvert, R., Jaeger, M., \& Jones, R. (1990). A visual representation and the control of manual aiming movements. Journal of Motor Behavior, 22, 327-346.

Elliott, D., Chua, R., \& Pollock, B. J. (1994). The influence of intermittent vision on manual aiming. Acta Psychologica, 85, 1-13.

Gegenfurtner, K. R., \& Sperling, G. (1993). Information transfer in iconic memory experiments. Journal of Experimental Psychology. Human Perception and Performance, 19, 845-866. doi:10.1037/ 0096-1523.19.4.845

Hoffman, J. E. (1979). A two-stage model of visual search. Perception \& Psychophysics, 25, 319-327.

Karni, A., \& Sagi, D. (1993). The time course of learning a visual skill. Nature, 365, 250-252.

Klingberg, T. (2010). Training and plasticity of working memory. Trends in Cognitive Sciences, 14, 317-324.

Long, G. M. (1980). Iconic memory: A review and critique of the study of short-term visual storage. Psychological Bulletin, 88, 785-820. doi:10.1037/0033-2909.88.3.785

Lu, Z.-L., Neuse, J., Madigan, S., \& Dosher, B. A. (2005). Fast decay of iconic memory in observers with mild cognitive impairments. Proceedings of the National Academy of Sciences, 102, 1797-1802.

Lyons, J., Fontaine, R., \& Elliot, D. (1997). I lost it in the lights: The effects of predictable and variable intermittent vision on unimanual catching. Journal of Motor Behavior, 29, 113-118.

Mednick, S. C., Makovski, T., Cai, D. J., \& Jiang, Y. V. (2009). Sleep and rest facilitate implicit memory in a visual search task. Vision Research, 49, 2557-2565.

Neisser, U. (1967). Cognitive psychology. Englewood Cliffs, NJ: Prentice Hall.

Olivier, I., Weeks, D. J., Lyons, J., Ricker, K. L., \& Elliott, D. (1998). Monocular and binocular vision in one-hand ball catching: Interocular integration. Journal of Motor Behavior, 30, 343-351.

Robertson, S., Collins, J., Elliott, D., \& Starkes, J. (1994). The influence of skill and intermittent vision on dynamic balance. Journal of Motor Behavior, 26, 333-339.

Savion-Lemieux, T., \& Penhune, V. B. (2005). The effects of practice and delay on motor skill learning and retention. Experimental Brain Research, 161, 423-431.

Scott, L. S., Tanaka, J. W., Sheinberg, D. L., \& Curran, T. (2008). The role of category learning in the acquisition and retention of perceptual expertise: A behavioral and neurophysiological study. Brain Research, 1210, 204-215.

Senders, J. W., Kristofferson, A. B., Levison, W. H., Dietrich, C. W., \& Ward, J. L. (1967). The attentional demand of automobile driving. Highway Research Record, 195, 15-33.

Smith, T. Q., \& Mitroff, S. R. (under review). Stroboscopic training enhances anticipatory timing.

Sperling, G. (1960). The information available in brief visual presentation. Psychological Monographs, 74(11, Whole No. 498), 129.

Webster, M. A., Mizokami, Y., \& Webster, S. M. (2007). Seasonal variations in the color statistics of natural images. Network: Computation in Neural Systems, 18, 213-233.

Westerberg, H., Jacobaeus, H., Hirvikoski, T., Clevberger, P., Ostensson, M. L., Bartfai, A., \& Klingberg, T. (2007). Computerized working memory training after stroke-A pilot study. Brain Injury, 21, 2129.

Wickens, T. D. (2001). Elementary signal detection theory. New York, NY: Oxford University Press. 